\title{
Nonrelativistic limits of colored gravity in three dimensions
}

\author{
Euihun Joung ${ }^{*}$ and Wenliang $\mathrm{Li}^{\dagger}$ \\ Department of Physics and Research Institute of Basic Science, Kyung Hee University, Seoul 02447, Korea
}

(Received 13 March 2018; published 29 May 2018)

\begin{abstract}
The three-dimensional nonrelativistic isometry algebras, namely Galilei and Newton-Hooke algebras, are known to admit double central extensions, which allows for nondegenerate bilinear forms hence for action principles through Chern-Simons formulation. In three-dimensional colored gravity, the same central extension helps the theory evade the multigraviton no-go theorems by enlarging the color-decorated isometry algebra. We investigate the nonrelativistic limits of three-dimensional colored gravity in terms of generalized İnönü-Wigner contractions.
\end{abstract}

DOI: 10.1103/PhysRevD.97.105020

\section{INTRODUCTION}

In the nonrelativistic limit, Einstein's general relativity reduces to Newtonian gravity. In analogy to EinsteinCartan gravity, Newtonian gravity has a coordinateindependent formulation in terms of frame fields, namely, Newton-Cartan gravity [1-5]. Since Einstein-Cartan gravity can be obtained by gauging the relativistic Poincare algebra, one may attempt to derive Newton-Cartan gravity by gauging a nonrelativistic symmetry algebra. The first candidate algebra would be the Galilei algebra $\mathfrak{g}_{0}$, which is the nonrelativistic limit of the Poincare algebra. However, it turns out that one instead needs to consider the Bargmann algebra $\mathfrak{g}_{0}^{+}$[6], which is an extension of the Galilei algebra with a central generator $M$ called "mass." This mass generator is related to an additional $U(1)$ gauge field in Newton-Cartan gravity. If we introduce a nonzero cosmological constant, then the counterparts of the Galilei algebra $\mathfrak{g}_{0}$ and the Bargmann algebra $\mathfrak{g}_{0}^{+}$are the Newton-Hooke algebra $\mathfrak{g}_{\Lambda}$ and the extended Newton-Hooke algebra $\mathfrak{g}_{\Lambda}^{+}$.

Since the equations of motion of Newton-Cartan gravity can be obtained by gauging the Bargmann algebra $\mathfrak{g}_{0}^{+}$[7], Newton-Cartan gravity can be considered as a gauge theory of the Bargmann algebra. Another way to derive NewtonCartan gravity is to consider a limit of Einstein-Cartan gravity mimicking the İnönü-Wigner contraction of the relativistic algebra [8]. However, in general spacetime dimensions, these equations of motion do not have a simple action principle.

\footnotetext{
*euihun.joung@khu.ac.kr

†lii.wenliang@gmail.com
}

Published by the American Physical Society under the terms of the Creative Commons Attribution 4.0 International license. Further distribution of this work must maintain attribution to the author(s) and the published article's title, journal citation, and DOI. Funded by SCOAP.
In three dimensions, the Bargmann algebra $\mathfrak{g}_{0}$ admits a second central extension by the "spin" generator $S$ [9-12]. ${ }^{1}$ The resulting algebra is referred to as the extended Bargmann algebra $\mathfrak{g}_{0}^{++}$. In parallel, in the cosmological case, the three-dimensional Newton-Hooke algebra admits double extensions. We refer to this as the doubly extended Newton-Hooke (NH) algebra $\mathfrak{g}_{\Lambda}^{++}$. We summarize the zoo of three-dimensional nonrelativistic algebras in Table I. Note that the subscript $\Lambda$ denotes the cosmological constant and the superscript + or ++ means the central extension by $M$ or $M, S$. Because of the second central extension, both $\mathfrak{g}_{0}^{++}$and $\mathfrak{g}_{\Lambda}^{++}$have nondegenerate invariant bilinear forms $[15,16]$. Consequently, the corresponding theories admit action principles through Chern-Simons formulation. These nonrelativistic gravity theories are sometimes called extended Bargmann gravity and extended NH gravity, respectively. Along the same lines, the nonrelativistic Chern-Simons theories admit various extensions according to the symmetry algebras [17-19].

The presence of two central generators $M$ and $S$ are hence crucial to the action principle of three-dimensional nonrelativistic gravities in the frame formulation. In fact, as we shall show later, these doubly extended nonrelativistic algebras can be obtained from the relativistic ones with two $u(1)$ generators after suitable contractions (see also Refs. $[17,20])$. For instance, in anti-de Sitter (AdS), the relativistic isometry algebra with two $u(1)$ 's,

$$
\begin{aligned}
& s o(2,2) \oplus u(1) \oplus u(1) \\
& \quad=(\operatorname{sl}(2, \mathbb{R}) \oplus u(1)) \oplus(\operatorname{sl}(2, \mathbb{R}) \oplus u(1)),
\end{aligned}
$$

contracts to the doubly extended $\mathrm{NH} \mathfrak{g}_{\Lambda}^{++}$under a suitable scaling limit. In fact, both the relativistic and nonrelativistic

\footnotetext{
${ }^{1}$ In Refs. [13,14], the second central extension is related to the noncommutativity of the particle's position coordinates.
} 
TABLE I. Three-dimensional nonrelativistic isometry algebras.

\begin{tabular}{lcc}
\hline \hline Extension & Flat & (Anti-)de Sitter \\
\hline None & Galilei $\mathfrak{g}_{0}$ & NH $\mathfrak{g}_{\Lambda}$ \\
$M$ & Bargmann $\mathfrak{g}_{0}^{+}$ & Extended NH $\mathfrak{g}_{\Lambda}^{+}$ \\
$M, S$ & Extended Bargmann $\mathfrak{g}_{0}^{++}$ & Doubly extended NH $\mathfrak{g}_{\Lambda}^{++}$ \\
\hline \hline
\end{tabular}

algebras can be split into left- and right-moving sectors, so the contraction can be performed in each sector. After the contraction, the $u(1)$ generators become central elements, and the resulting algebra cannot be arranged as a direct sum anymore. This is the key mechanism for a nondegenerate bilinear form-and hence for the action principle.

We notice that another peculiar utility of $u(1)$ can be found in a different extension of three-dimensional gravity, namely, the colored gravity, which has been studied recently in Refs. [21-23]. There exists a no-go result [24] for theories of multiple gravitons, ${ }^{2}$ but in three dimensions, it can be evaded ${ }^{3}$ by extending the isometry algebra $\operatorname{so}(2,2)$ with two $u(1)$ generators. It is remarkable that the resolution of the problems in the nonrelativistic limit and color decoration are the same: replace $s l(2, \mathbb{R})$ by $s l(2, \mathbb{R}) \oplus u(1)$. This observation leads us to the following question: does the three-dimensional colored gravity have a natural nonrelativistic limit?

Let us explain a few more details about the colored gravity. It can be constructed in the Chern-Simons formulation with an appropriate choice of gauge algebra. For the multigraviton interpretation, this algebra should contain multiple copies of isometry generators, hence is a direct product of the isometry so $(2,2)$ and the internal symmetry. Usually, the direct product of two Lie algebras does not define a Lie algebra, but the case of two associative algebras does so. The internal symmetry can be taken as $U(N)$, which obviously has an associative structure. About the isometry algebra, we can extend the Lie algebra $\operatorname{sl}(2, \mathbb{R}) \oplus \operatorname{sl}(2, \mathbb{R})$ to an associative algebra $g l(2, \mathbb{R}) \oplus \operatorname{gl}(2, \mathbb{R})$ by adding two copies of $u(1)$. We refer to the internal symmetry as "color symmetry" for a reason that will become clear later, and the resulting gravity theory will be correspondingly referred to as "colored gravity." The colored gravity has several interesting features:

(i) It has a nontrivial potential with many saddle points corresponding to the (A)dS vacua of different

\footnotetext{
${ }^{2}$ Multigraviton theories are obtained by gauging the tensor product of the spacetime isometry and an internal, associative, commutative algebra equipped with a nondegenerate invariant norm. See Ref. [25] for a review and Ref. [26] for an extension to the conformal (super)gravities. In these results, no additional fields besides the graviton (multiplet) are introduced, as opposed to Refs. [21-23].

${ }^{3}$ The first exception to the no-go result in three dimensions was found in Ref. [27], in which an "exotic" multigraviton theory was constructed by allowing for a $P T$-noninvariant interaction. Interestingly, such an exotic interaction can be obtained by a contraction of the usual Einstein gravity after a field redefinition.
}

cosmological constants. These vacua can be classified according to how much the internal symmetry is preserved.

(ii) Around the color singlet vacuum, the theory has one graviton, $\left(N^{2}-1\right)$ massless spin-2 fields charged under the color symmetry, and a $U(N) \times U(N)$ Chern-Simons gauge field. The color nonsinglet spin-2 fields are strongly interacting for large $N$.

(iii) Around a color symmetry-breaking background, a Higgs-like mechanism takes place, and one finds partially massless spin-2 fields [28,29] appearing in the spectrum.

The organization of the paper is as follows. In Sec. II, we present a concise overview of several aspects of the threedimensional nonrelativistic algebras. In Sec. III, we derive the nonrelativistic colored-gravity algebras from the İnönüWigner contractions of the relativistic ones. In Sec. IV, we construct the actions of nonrelativistic colored gravities using the Chern-Simons formulation. In Sec. V, we conclude the work with a brief summary. The Appendix includes technical details and additional materials.

\section{NONRELATIVISTIC ALGEBRAS AND THEIR BILINEAR FORMS}

\section{A. Various nonrelativistic algebras in three dimensions}

We will give a concise review of various threedimensional nonrelativistic algebras: the Galilei, Bargmann, extended Bargmann, $\mathrm{NH}$, extended $\mathrm{NH}$, and doubly extended $\mathrm{NH}$ algebras, which have been briefly discussed in the Introduction.

\section{Galilei algebra $\mathfrak{g}_{0}$}

The three-dimensional Galilei algebra is an eightdimensional Lie algebra with the nontrivial Lie brackets:

$$
\left[J, G_{i}\right]=2 \epsilon_{i j} G_{j}, \quad\left[J, P_{i}\right]=2 \epsilon_{i j} P_{j}, \quad\left[H, G_{i}\right]=2 \epsilon_{i j} P_{j}
$$

Here, $H, P_{i}, J$, and $G_{i}$ are the generators of time translations, spatial translations, spatial rotations, and Galilean boosts, respectively. The Levi-Cività symbol $\epsilon_{i j}$ is defined as

$$
\epsilon_{12}=-\epsilon_{21}=1, \quad \epsilon_{11}=\epsilon_{22}=0 .
$$

Remark that, in higher dimensions, there exist nonzero brackets between two spatial rotation generators.

\section{Newton-Hooke algebra $\mathfrak{g}_{\Lambda}$}

When the cosmological constant $\Lambda$ is turned on, the Galilei algebra is deformed to the so-called Newton-Hooke algebra. As a result, we have an additional set of Lie brackets, 


$$
\left[H, P_{i}\right]=-2 \Lambda \epsilon_{i j} G_{j},
$$

besides those in Eq. (2).

\section{Bargmann algebra $\mathrm{g}_{0}^{+}$and extended NH algebra $\mathfrak{g}_{\Lambda}^{+}$}

In general dimensions, both the Galilean and $\mathrm{NH}$ algebras can be centrally extended by the mass generator $M{ }^{4}$ The resulting algebras are known as the Bargmann algebra and the extended $\mathrm{NH}$ algebra, respectively. In three dimensions, the additional Lie brackets are

$$
\left[G_{i}, P_{j}\right]=2 \epsilon_{i j} M .
$$

\section{Extended Bargmann algebra $\mathfrak{g}_{0}^{++}$and doubly extended $\mathrm{NH}$ algebra $\mathrm{g}_{\Lambda}^{++}$}

In three dimensions, the Bargmann and extended $\mathrm{NH}$ algebras admit a second central extension by the spin generator $S .^{5}$ The extended Bargmann algebra, or equivalently the doubly extended Galilei algebra, has one more set of Lie brackets,

$$
\left[G_{i}, G_{j}\right]=2 \epsilon_{i j} S .
$$

In the doubly extended Newton-Hooke algebra, the additional Lie brackets are (6) and

$$
\left[P_{i}, P_{j}\right]=-2 \Lambda \epsilon_{i j} S .
$$

All the nonrelativistic algebras presented above can be obtained from the most general one, the doubly extended $\mathrm{NH}$ algebra, by various İnönü-Wigner contractions. The cosmological constant-dependent terms can be removed by the usual İnönü-Wigner contraction. The mass and spin generators only appear in the right-hand sides of the Lie brackets and hence can be eliminated by a suitable contraction: rescale them first by a contraction parameter, and then send the parameter to zero.

\section{B. Nonrelativistic limits as contractions}

It is useful to realize these nonrelativistic limits as contractions. In fact, the three-dimensional relativistic isometry algebra with a nonzero cosmological constant and two additional $u(1)$ generators can contract to the doubly extended $\mathrm{NH}$ algebra (hence, all nonrelativistic algebras discussed in Sec. II A). Let us provide more details

\footnotetext{
${ }^{4}$ In a relativistic theory, the energy of a particle in the rest frame is proportional to the rest mass. However, in a nonrelativistic theory, spacetime symmetries do not require energy-mass equivalence, so we can consider an independent generator for mass in the form of a central extension. Intuitively speaking, the energymass relation is lost in the large speed-of-light limit.

${ }^{5}$ To interpret $S$ as a spin generator is subtle. See Refs. [30,31] for more details.
}

on this point. The relativistic isometry algebra in three dimensions has the Lie brackets

$$
\begin{aligned}
{\left[\mathcal{J}_{a}, \mathcal{J}_{b}\right] } & =2 \epsilon_{a b}{ }^{c} \mathcal{J}_{c}, \quad\left[\mathcal{J}_{a}, \mathcal{P}_{b}\right]=2 \epsilon_{a b}{ }^{c} \mathcal{P}_{c}, \\
{\left[\mathcal{P}_{a}, \mathcal{P}_{b}\right] } & =-2 \Lambda \epsilon_{a b}{ }^{c} \mathcal{J}_{c},
\end{aligned}
$$

where $\mathcal{P}_{a}$ and $\mathcal{J}_{a}$ are the translation and Lorentz generators and $\Lambda$ is the cosmological constant.

\section{Contraction to Newton-Hooke algebra}

For an illustration, let us see how the relativistic isometry algebra (8) contracts to the Newton-Hooke algebra $\mathfrak{g}_{\Lambda}$. We first redefine the generators using the parameter $c$ as

$$
G_{i}=\frac{1}{c} \mathcal{J}_{i}, \quad P_{i}=\frac{1}{c} \mathcal{P}_{i}
$$

and

$$
J=\mathcal{J}_{0}, \quad H=\mathcal{P}_{0}
$$

then derive their Lie brackets. Notice that the temporal components scale differently in $c$ from the spatial ones. The İnönü-Wigner contraction is performed in the $c \rightarrow \infty$ limit, which effectively removes certain terms in the structure constants. One can easily check that the resulting algebra is the $\mathrm{NH}$ algebra $\mathfrak{g}_{\Lambda}$. Hence, one can view this contraction as the nonrelativistic limit and interpret the parameter $c$ as the speed of light.

\section{Contraction to doubly extended Newton-Hooke algebra}

Let us now show how the doubly extended $\mathrm{NH}$ algebra $\mathfrak{g}_{\Lambda}^{++}$can be derived by a contraction. As the algebra $\mathfrak{g}_{\Lambda}^{++}$has dimension 8 due to the central generators $M$ and $S$, it cannot be obtained as a contraction of the relativistic isometry algebra, which has dimension 6. To fix the mismatch between the numbers of generators, we need to include two additional $u(1)$ generators. We refer to these generators as $\mathcal{Z}_{\mathcal{P}}$ and $\mathcal{Z}_{\mathcal{J}}$. In terms of them, we change the definitions of $H$ and $J$ to

$$
J=\mathcal{Z}_{\mathcal{J}}+\mathcal{J}_{0}, \quad H=\mathcal{Z}_{\mathcal{P}}+\mathcal{P}_{0}
$$

but keep those of $P_{i}$ and $G_{i}$ the same as (9). The mass and spin generators are given by the other combinations with different rescalings,

$$
S=\frac{\mathcal{Z}_{\mathcal{J}}-\mathcal{J}_{0}}{2 c^{2}}, \quad M=\frac{\mathcal{Z}_{\mathcal{P}}-\mathcal{P}_{0}}{2 c^{2}} .
$$

With these definitions, one can successfully reproduce the doubly extended $\mathrm{NH}$ algebra $\mathfrak{g}_{\Lambda}^{++}$by taking the $c \rightarrow \infty$ limit. Note that in Eqs. (11) and (12) the mixings with $\mathcal{Z}_{\mathcal{P}}$ 
and $\mathcal{Z}_{\mathcal{J}}$ are independent, and hence the corresponding central extensions by $M$ and $S$ are also independent.

\section{Chiral decomposition}

When $\Lambda \neq 0$, the relativistic isometry algebra can be decomposed into two parts,

$$
\begin{aligned}
& \mathcal{L}_{a}=\frac{1}{2}\left(\mathcal{J}_{a}+\frac{1}{\sqrt{-\Lambda}} \mathcal{P}_{a}\right), \\
& \tilde{\mathcal{L}}_{a}=\frac{1}{2}\left(\mathcal{J}_{a}-\frac{1}{\sqrt{-\Lambda}} \mathcal{P}_{a}\right),
\end{aligned}
$$

and each of them forms an $\operatorname{sl}(2)$ :

$$
\left[\mathcal{L}_{a}, \mathcal{L}_{b}\right]=2 \epsilon_{a b}{ }^{c} \mathcal{L}_{c}, \quad\left[\tilde{\mathcal{L}}_{a}, \tilde{\mathcal{L}}_{b}\right]=2 \epsilon_{a b}^{c} \tilde{\mathcal{L}}_{c} .
$$

Accordingly, it is natural to decompose the $u(1) \oplus u(1)$ part as

$$
\begin{aligned}
& \mathcal{Z}=\frac{1}{2}\left(\mathcal{Z}_{\mathcal{J}}+\frac{1}{\sqrt{-\Lambda}} \mathcal{Z}_{\mathcal{P}}\right), \\
& \tilde{\mathcal{Z}}=\frac{1}{2}\left(\mathcal{Z}_{\mathcal{J}}-\frac{1}{\sqrt{-\Lambda}} \mathcal{Z}_{\mathcal{P}}\right) .
\end{aligned}
$$

For the nonrelativistic limit $c \rightarrow \infty$, one can check that the redefinitions (9), (11), and (12) are compatible with the chiral decomposition. More precisely, with the redefinitions,

$$
\begin{aligned}
K=\mathcal{Z}+\mathcal{L}_{0}, & L_{i}=\frac{1}{c} \mathcal{L}_{i}, & Z=\frac{\mathcal{Z}-\mathcal{L}_{0}}{2 c^{2}}, \\
\tilde{K}=\tilde{\mathcal{Z}}+\tilde{\mathcal{L}}_{0}, & \tilde{L}_{i}=\frac{1}{c} \tilde{\mathcal{L}}_{i}, & \tilde{Z}=\frac{\tilde{\mathcal{Z}}-\tilde{\mathcal{L}}_{0}}{2 c^{2}},
\end{aligned}
$$

the two copies of the relativistic chiral algebra $\operatorname{sl}(2) \oplus u(1)$ contract to the nonrelativistic chiral algebras

$$
\begin{array}{ll}
{\left[K, L_{i}\right]=2 \epsilon_{i j} L_{j}, \quad\left[L_{i}, L_{j}\right]=2 \epsilon_{i j} Z,} \\
{\left[\tilde{K}, \tilde{L}_{i}\right]=2 \epsilon_{i j} \tilde{L}_{j},} & {\left[\tilde{L}_{i}, \tilde{L}_{j}\right]=2 \epsilon_{i j} \tilde{Z},}
\end{array}
$$

where $Z$ and $\tilde{Z}$ are the centers of the chiral algebras. The chiral subalgebra $\mathfrak{h}$, spanned by $K, L_{i}$, and $Z$, can be viewed as a central extension of the two-dimensional Euclidean algebra in which $K$ and $L_{i}$ can be interpreted as twodimensional translation and rotation generators, respectively. One can equally interpret $\mathfrak{h}$ as a harmonic oscillator, in which $L_{1} \pm i L_{2}$ are creation/annihilation or position/ momentum operators, $K$ is the Hamiltonian, and $Z$ is the Plank constant $\hbar .^{6}$

\footnotetext{
${ }^{6}$ The same algebra can be obtained from the subalgebra of Virasoro algebra spanned by $L_{m} / m^{3 / 2}, L_{-m} / m^{3 / 2}$, and $L_{0} / m$ (here, $L_{m}$ are the standard generators of Virasoro algebra) in the $m \rightarrow \infty$ limit. The Virasoro central charge $c$ becomes the central element $Z$.
}

The doubly extended $\mathrm{NH}$ algebra $\mathfrak{g}_{\Lambda}^{++}$can be directly decomposed into two copies of the chiral algebra $\mathfrak{h}$ and $\tilde{\mathfrak{h}}$,

$$
\mathfrak{g}_{\Lambda}^{++}=\mathfrak{h} \oplus \tilde{\mathfrak{h}},
$$

where the explicit decomposition reads

$$
\begin{aligned}
G_{i} & =L_{i}+\tilde{L}_{i}, \quad J=K+\tilde{K}, \quad S=Z+\tilde{Z}, \\
P_{i} & =\sqrt{-\Lambda}\left(L_{i}-\tilde{L}_{i}\right), \quad H=\sqrt{-\Lambda}(K-\tilde{K}), \\
M & =\sqrt{-\Lambda}(Z-\tilde{Z}) .
\end{aligned}
$$

\section{Invariant bilinear form}

One may attempt to use the nonrelativistic algebras in Sec. II A to construct nonrelativistic gravity theories. In three dimensions, as in the relativistic case, one can rely on the Chern-Simons formulation in which nondegeneracy of the bilinear form is important for the dynamics of the resulting theory. It turns out that, among the algebras that we have considered, only those with double extensions by $M$ and $J$ admit a nondegenerate bilinear form. Below, we focus on the doubly extended $\mathrm{NH}$ algebra and discuss its bilinear form.

Since the doubly extended $\mathrm{NH}$ algebra $\mathfrak{g}_{\Lambda}^{++}$admits the decomposition (20), we can consider the invariant bilinear form $\langle\cdot, \cdot\rangle$ for each chiral sector. The nondiagonal parts are the $2 \times 2$ symmetric matrices,

$$
B=\left(\begin{array}{cc}
\langle K, K\rangle & \langle Z, K\rangle \\
\langle K, Z\rangle & \langle Z, Z\rangle
\end{array}\right), \quad \tilde{B}=\left(\begin{array}{cc}
\langle\tilde{K}, \tilde{K}\rangle & \langle\tilde{Z}, \tilde{K}\rangle \\
\langle\tilde{K}, \tilde{Z}\rangle & \langle\tilde{Z}, \tilde{Z}\rangle
\end{array}\right) .
$$

Because of the rotational symmetry, the diagonal part is fixed as

$$
\left\langle L_{i}, L_{j}\right\rangle=\ell \delta_{i j}, \quad\left\langle\tilde{L}_{i}, \tilde{L}_{j}\right\rangle=\tilde{\ell} \delta_{i j}
$$

with undetermined constants $\ell$ and $\tilde{\ell}$, while the other bilinear forms $\left\langle L_{i}, K\right\rangle,\left\langle L_{i}, Z\right\rangle$, and their tilded counterparts vanish. Symmetry also determines the matrices $B$ and $\tilde{B}$ to be

$$
B=\left(\begin{array}{cc}
\beta & \ell \\
\ell & 0
\end{array}\right), \quad \tilde{B}=\left(\begin{array}{cc}
\tilde{\beta} & \tilde{\ell} \\
\tilde{\ell} & 0
\end{array}\right)
$$

where $\beta$ and $\tilde{\beta}$ are additional allowed constants. The nondegeneracy of the bilinear form requires $\ell \neq 0$ and $\tilde{\ell} \neq 0$, but $\beta$ and $\tilde{\beta}$ are left unconstrained.

The bilinear form of the doubly extended $\mathrm{NH}$ algebra (21) is given by those of the left and right chiral algebras $\mathfrak{h}$ and $\tilde{\mathfrak{h}}$, together with possible cross-terms. However, one can show that the only cross-term compatible with symmetry is 


$$
\langle K, \tilde{K}\rangle=\gamma .
$$

In the end, the invariant bilinear form of $\mathfrak{g}_{\Lambda}^{++}$has five undetermined constants:

$$
\ell, \tilde{\ell}, \beta, \tilde{\beta}, \gamma \text {. }
$$

The number of free parameters is consistent with the analysis of Ref. [18].

As in the usual relativistic Chern-Simons gravity, we set $\ell$ and $\tilde{\ell}$ as

$$
\ell=-\tilde{\ell}=\frac{1}{2 \sqrt{-\Lambda}}
$$

so the spatial part takes the standard form. The nonvanishing components of the bilinear form are

$$
\langle J, M\rangle=\langle S, H\rangle=1, \quad\left\langle P_{i}, G_{j}\right\rangle=\delta_{i j},
$$

and

$$
\left(\begin{array}{cc}
\langle H, H\rangle & \langle H, J\rangle \\
\langle J, H\rangle & \langle J, J\rangle
\end{array}\right)=\left(\begin{array}{cc}
-\Lambda(\beta+\tilde{\beta}-2 \gamma) & \sqrt{-\Lambda}(\beta-\tilde{\beta}) \\
\sqrt{-\Lambda}(\beta-\tilde{\beta}) & \beta+\tilde{\beta}+2 \gamma
\end{array}\right) .
$$

The above symmetric matrix remains completely arbitrary.

The bilinear form that we have derived using symmetry can also be obtained from the relativistic one by a contraction. To begin with, let us focus on the chiral sector. The bilinear form of $\mathfrak{h}=\operatorname{sl}(2) \oplus u(1)$ is unique for each of the $\operatorname{sl}(2)$ and $u(1)$ parts, up to overall factors:

$$
\begin{gathered}
\langle\mathcal{Z}, \mathcal{Z}\rangle=\zeta, \quad\langle\tilde{\mathcal{Z}}, \tilde{\mathcal{Z}}\rangle=\tilde{\zeta} \\
\left\langle\mathcal{L}_{a}, \mathcal{L}_{b}\right\rangle=\lambda \eta_{a b}, \quad\left\langle\tilde{\mathcal{L}}_{a}, \tilde{\mathcal{L}}_{b}\right\rangle=\tilde{\lambda} \eta_{a b} .
\end{gathered}
$$

Using Eqs. (16) and (17), these give the bilinear form for the rescaled generators as

$$
\begin{gathered}
\left\langle L_{i}, L_{j}\right\rangle=\frac{\lambda}{c^{2}} \delta_{i j}, \quad\left\langle\tilde{L}_{i}, \tilde{L}_{j}\right\rangle=\frac{\tilde{\lambda}}{c^{2}} \delta_{i j}, \\
B=\left(\begin{array}{cc}
\zeta-\lambda & (\zeta+\lambda) /\left(2 c^{2}\right) \\
(\zeta+\lambda) /\left(2 c^{2}\right) & (\zeta-\lambda) /\left(4 c^{4}\right)
\end{array}\right), \\
\tilde{B}=\left(\begin{array}{cc}
\tilde{\zeta}-\tilde{\lambda} & (\tilde{\zeta}+\tilde{\lambda}) /\left(2 c^{2}\right) \\
(\tilde{\zeta}+\tilde{\lambda}) /\left(2 c^{2}\right) & (\tilde{\zeta}-\tilde{\lambda}) /\left(4 c^{4}\right)
\end{array}\right) .
\end{gathered}
$$

Hence, by choosing

$$
\begin{array}{ll}
\zeta=c^{2} \ell+\beta / 2, & \tilde{\zeta}=c^{2} \tilde{\ell}+\tilde{\beta} / 2, \\
\lambda=c^{2} \ell-\beta / 2, & \tilde{\lambda}=c^{2} \tilde{\ell}-\tilde{\beta} / 2,
\end{array}
$$

we recover the bilinear form (24) in the contraction limit. Then, we consider the brackets of the left and right sectors. We have the freedom to introduce the cross-term,

$$
\langle\mathcal{Z}, \tilde{Z}\rangle=\gamma
$$

which leads to Eq. (25) after the contraction.

We have seen that all the nonrelativistic algebras and their bilinear forms can be obtained from the relativistic one by contractions. In addition, it is also sufficient to consider the chiral sector $\operatorname{sl}(2) \oplus u(1)$. At the level of Lie algebra, there is no relation between $s l(2)$ and $u(1)$, and hence their bilinear forms (30) are also independent.

On the other hand, one simple way to introduce an invariant bilinear form is through an invariant linear form, i.e., trace:

$$
\langle X, Y\rangle=\operatorname{Tr}(X Y) .
$$

A well-defined trace requires an associative product between $X$ and $Y$. This can be done by representing $X$ and $Y$ as matrices. But the price to pay is that we have to work with a larger space because the associative products of the original elements will generate additional elements. The enlargement can be minimized by choosing the smallest representation. For $s l(2)$, the smallest representation is the fundamental representation, which is two dimensional and requires only one more element, i.e., the identity, to have the associative structure. The associative product of the relativistic chiral algebra reads

$\mathcal{L}_{a} \mathcal{L}_{b}=\eta_{a b} \mathcal{I}+\epsilon_{a b}{ }^{c} \mathcal{L}_{c}, \quad \tilde{\mathcal{L}}_{a} \tilde{\mathcal{L}}_{b}=\eta_{a b} \tilde{\mathcal{I}}+\epsilon_{a b}{ }^{c} \tilde{\mathcal{L}}_{c}$,

and we recover the bilinear form (31) using

$\operatorname{Tr}(\mathcal{I})=\lambda, \quad \operatorname{Tr}(\tilde{\mathcal{I}})=\tilde{\lambda}, \quad \operatorname{Tr}\left(\mathcal{L}_{a}\right)=\operatorname{Tr}\left(\tilde{\mathcal{L}}_{a}\right)=0$.

There is no a priori reason to identify the identities $\mathcal{I}$ and $\tilde{\mathcal{I}}$ with the $u(1)$ generators $\mathcal{Z}$ and $\tilde{\mathcal{Z}}$. However, if they coincide up to some factors $z(c)$ and $\tilde{z}(c)$, the coefficients $\lambda, \tilde{\lambda}$ and $\zeta, \tilde{\zeta}$ in Eqs. (30) and (31) will be related as

$$
\begin{aligned}
& \mathcal{Z}=z(c) \mathcal{I} \Rightarrow \zeta=z^{2}(c) \lambda, \\
& \tilde{\mathcal{Z}}=\tilde{z}(c) \tilde{\mathcal{I}} \Rightarrow \tilde{\zeta}=\tilde{z}^{2}(c) \tilde{\lambda} .
\end{aligned}
$$

These relations together with Eq. (34) fix the form of the functions $z(c)$ and $\tilde{z}(c)$ as

$$
z^{2}(c)=\frac{c^{2} \ell+\beta / 2}{c^{2} \ell-\beta / 2}, \quad \tilde{z}^{2}(c)=\frac{c^{2} \tilde{\ell}+\tilde{\beta} / 2}{c^{2} \tilde{\ell}-\tilde{\beta} / 2} .
$$

Therefore, we fully recover the nonrelativistic algebras $\mathfrak{h}$ and $\tilde{\mathfrak{h}}$ and their bilinear forms using the associative structure of the relativistic counterpart, $g l(2)$. As we 
mentioned in the Introduction, the associative structure of the isometry algebras plays a central role in the color decoration of three-dimensional gravity. From this observation, we are driven to look more closely the possible interplay between nonrelativistic and color deformations of gravity in three dimensions.

\section{COLORED GRAVITY AND ITS NONRELATIVISTIC LIMITS}

If a theory contains more than one graviton and they are related by internal symmetry, the global symmetry should be extended from the usual isometry $\mathfrak{g}_{i}$ to a color-decorated one $\mathfrak{g}_{i} \otimes \mathfrak{g}_{c}$, where $\mathfrak{g}_{c}$ is a finite vector space generated by the color label. This leads to severe algebraic constraints: there is only trivial possibility if we assume the colordecorated algebra does not include additional generators, which means extra fields are necessary $[21,22]$. Even if we find a good candidate of colored-gravity algebras by including a reasonable amount of additional fields, the construction of equations of motion or/and actions is not guaranteed to be straightforward. In three dimensions, the situation is much simpler. First, thanks to the ChernSimons formulation, we obtain the action directly from a given algebra. Second, a color-decorated algebra can be constructed by extending the isometry algebra with only two additional $u(1)$ generators.

Technically speaking, we want to find a Lie algebra structure on the tensor-product vector space $\mathfrak{g}_{i} \otimes \mathfrak{g}_{c}$. The Lie brackets between two elements of the above space may be written as

$$
\begin{aligned}
{\left[M_{X} \otimes \mathcal{T}^{I}, M_{Y} \otimes \mathcal{T}^{J}\right]=} & \frac{1}{2}\left[M_{X}, M_{Y}\right] \otimes\left\{\mathcal{T}^{I}, \mathcal{T}^{J}\right\} \\
& +\frac{1}{2}\left\{M_{X}, M_{Y}\right\} \otimes\left[\mathcal{T}^{I}, \mathcal{T}^{J}\right],
\end{aligned}
$$

where $M_{X}$ and $M_{Y}$ belong to $\mathfrak{g}_{i}$, whereas $\mathcal{T}^{I}$ and $\mathcal{T}^{J}$ belong to $\mathfrak{g}_{c}$. Note that we have used both the commutator and anticommutator of the generators of each space. Therefore, to have well-defined Lie brackets (42), we should begin with two associative algebras $\mathfrak{g}_{i}$ and $\mathfrak{g}_{c}$. For the color algebra $\mathfrak{g}_{c}$, we use the matrix algebra $u(N){ }^{7}$ For the isometry algebra $\mathfrak{g}_{i}$, the minimal choice is $g l(2) \oplus g l(2)$. This is precisely the same as the setting (1) for the doubly extended $\mathrm{NH}$ algebra.

\section{A. Chiral sector}

Let us start from the nonrelativistic limits of one chiral sector of the colored-gravity algebra. It is generated by the generators,

\footnotetext{
${ }^{7}$ In principle, any finite-dimensional associative algebra can be used as the color algebra.
}

$$
\mathcal{Z}, \quad \mathcal{L}_{a}, \quad \mathcal{T}^{I}, \quad \mathcal{L}_{a}^{I},
$$

where $\mathcal{L}_{a}$ and $\mathcal{Z}$ form the original relativistic algebra $s l(2) \oplus u(1)=g l(2)$ and $\mathcal{T}^{I}$ are the $s u(N)$ generators:

$$
\left[\mathcal{T}^{I}, \mathcal{T}^{J}\right]=2 i{ }^{I J}{ }_{K} \mathcal{T}^{K} .
$$

The generators $\mathcal{L}_{a}^{I}$ are the color decoration of the spin-2 generators, and they transform covariantly under $\operatorname{sl}(2)$ and $\operatorname{su}(N)$,

$$
\left[\mathcal{L}_{a}, \mathcal{L}_{b}^{I}\right]=2 \epsilon_{a b}{ }^{c} \mathcal{L}_{c}^{I}, \quad\left[\mathcal{T}^{I}, \mathcal{L}_{a}^{J}\right]=2 i f^{I J}{ }_{K} \mathcal{L}_{a}^{K},
$$

but the Lie brackets between them are nontrivial:

$$
\left[\mathcal{L}_{a}^{I}, \mathcal{L}_{b}^{J}\right]=2 i \eta_{a b} f^{I J}{ }_{K} \mathcal{T}^{K}+2 \epsilon_{a b}{ }^{c}\left(\frac{1}{N} \delta^{I J} \mathcal{L}_{c}+g^{I J}{ }_{K} \mathcal{L}_{c}^{K}\right)
$$

In Eq. (46), we used the associative algebra of $u(N)$,

$$
\mathcal{T}^{I} \mathcal{T}^{J}=\frac{1}{N} \delta^{I J} I+g^{I J}{ }_{K} \mathcal{T}^{K}+i f^{I J}{ }_{K} \mathcal{T}^{K}
$$

where $g_{I J K}$ is totally symmetric and $f_{I J K}$ totally antisymmetric. We assign its bilinear form as

$$
\left\langle\mathcal{T}^{I}, \mathcal{T}^{J}\right\rangle=\operatorname{Tr}\left(\mathcal{T}^{I} \mathcal{T}^{J}\right)=\delta^{I J},
$$

where we used $\operatorname{Tr}(I)=N$ while $\operatorname{Tr}\left(\mathcal{T}^{I}\right)=0$.

We want to construct the nonrelativistic analogs of the above colored-gravity algebra, which we assume to have the same number of generators as the relativistic one. The nonrelativistic algebra is spanned by

$$
Z, \quad L_{i}, \quad K, \quad \mathcal{T}^{I}, \quad \boldsymbol{L}_{i}^{I}, \quad \boldsymbol{K}^{I} .
$$

Their Lie brackets depend on the nonrelativistic limits.

\section{Color decoration after contraction}

One of the possible ways to obtain the nonrelativistic colored-gravity algebra is by colored decorating the doubly extended $\mathrm{NH}$ algebra. For that, the latter algebra should admit an associative algebra structure. In the previous section, we have shown that it can be obtained from an associative relativistic algebra by a suitable contraction. However, we have not examined the full consistency of the associative structure of the resulting nonrelativistic algebra. An associative product can be split into commutators, which reproduce the Lie brackets, and anticommutators. Therefore, we have to check whether the contraction limit (16) is equally well defined for the anticommutators. From Eqs. (37), (39), and (41), most of the anticommutators are regular in the contraction limit: 


$$
\begin{aligned}
& \left\{K, L_{i}\right\}=2 L_{i}, \quad\{K, Z\}=2 Z, \quad\left\{L_{i}, L_{j}\right\}=2 \delta_{i j} Z, \\
& \left\{L_{i}, Z\right\}=0, \quad\{Z, Z\}=0 .
\end{aligned}
$$

However, before taking the contraction limit, the $\{K, K\}$ anticommutator reads

$$
\{K, K\}=2\left(K-2 c^{2} Z\right) .
$$

In the $c \rightarrow \infty$ limit, the anticommutator (51) becomes ill defined, so the corresponding associative product is problematic. The contraction of the $g l(2)$ gives a good Lie algebra structure and bilinear form or trace, but not the fully associative algebra structure.

This issue does not affect the Chern-Simons action of the doubly extended $\mathrm{NH}$ gravity. The reason we consider the anticommutator $\{K, K\}$ here is that from Eq. (42) it appears in the Lie bracket of its color-decorated counterparts, $\left[\boldsymbol{K}^{I}, \boldsymbol{K}^{J}\right]$. We can avoid the divergence by defining the colored generators as

$$
\boldsymbol{K}^{I}=\frac{1}{c} K \otimes \mathcal{T}^{I}, \quad Z^{I}=Z \otimes \mathcal{T}^{I},
$$

which leads to a regular commutator,

$$
\left[\boldsymbol{K}^{I}, \boldsymbol{K}^{J}\right]=-4 i f^{I J}{ }_{K} \boldsymbol{Z}^{K} .
$$

Concerning the color decoration of $L_{i}$ generators, we consider two options:

(i) In the first option, by defining them as

$$
\boldsymbol{L}_{i}^{I}=\frac{1}{c} L_{i} \otimes \mathcal{T}^{I}
$$

we have

$$
\left[\boldsymbol{K}^{I}, L_{i}\right]=\left[K, \boldsymbol{L}_{i}^{I}\right]=2 \epsilon_{i j} \boldsymbol{L}_{j}^{I}
$$

(ii) In the second option, with the definition

$$
\boldsymbol{L}_{i}^{I}=L_{i} \otimes \mathcal{T}^{I},
$$

we obtain different Lie brackets:

$$
\begin{aligned}
{\left[K, \boldsymbol{L}_{i}^{I}\right]=} & 2 \epsilon_{i j} \boldsymbol{L}_{j}^{I}, \quad\left[L_{i}, \boldsymbol{L}_{j}^{I}\right]=2 \epsilon_{i j} \boldsymbol{Z}^{I}, \\
{\left[\boldsymbol{L}_{i}^{I}, \boldsymbol{L}_{j}^{J}\right]=} & 2 \epsilon_{i j}\left(\frac{1}{N} \epsilon_{i j} \delta^{I J} Z+2 g^{I J}{ }_{K} \boldsymbol{Z}^{K}\right) \\
& +2 i \delta_{i j} f^{I J}{ }_{K} \boldsymbol{Z}^{K} .
\end{aligned}
$$

The rest of the Lie brackets vanish in both cases.

In the above paragraph, we obtained two nonrelativistic colored-gravity algebras with suitable contractions. However, it turns out that they do not have nondegenerate invariant bilinear form due to the additional contraction related to the color decoration. This problem can be fixed by considering the mixing between the two chiral algebras, which will be explained in Sec. III C. In the next subsection, we will introduce the generalized İnönü-Wigner contraction, the concept of which is crucial to the systematic construction of nonrelativistic algebras from the relativistic ones.

\section{B. Generalized İnönü-Wigner contraction}

İnönü-Wigner contraction [32] is a procedure for generating Lie algebras from a starting Lie algebra by taking certain limits of the group-contraction parameters. The contraction procedure involves singular changes of bases, so the contracted algebra is not isomorphic to the original algebra. If a Lie algebra $\mathfrak{g}$ has a subalgebra $\mathfrak{h}$, we can decompose $\mathfrak{g}$ into

$$
\mathfrak{g}=\mathfrak{h} \oplus \mathfrak{i} .
$$

We then rescale the generators of $\mathfrak{i}$ by a contraction parameter $c$ and introduce the new generators as

$$
\dot{\mathrm{i}}=\frac{1}{c} \dot{\mathrm{i}} .
$$

The Lie brackets become

$$
[\mathfrak{h}, \mathfrak{h}] \subset \mathfrak{h}, \quad[\mathfrak{h}, \mathfrak{\mathfrak { j }}] \subset \frac{1}{c} \mathfrak{h} \oplus \dot{\mathfrak{i}}, \quad[\mathfrak{i}, \mathfrak{j}] \subset \frac{1}{c^{2}} \mathfrak{h} \oplus \frac{1}{c} \dot{\mathfrak{j}} .
$$

In the singular limit where $c \rightarrow \infty$, we obtain a new Lie algebra,

$$
\mathcal{G}(\mathfrak{g})=\mathfrak{h} \oplus \dot{\mathfrak{j}},
$$

with the Lie brackets

$$
[\mathfrak{h}, \mathfrak{h}] \subset \mathfrak{h}, \quad[\mathfrak{h}, \mathfrak{j}] \subset \mathfrak{j}, \quad[\mathfrak{i}, \mathfrak{j}] \subset \varnothing,
$$

where $\mathrm{j}$ corresponds to an Abelian ideal. As an example, the Poincaré algebra can be obtained from the (A)dS algebra by sending the radius of curvature to infinity, and the translation generators in the Poincaré algebra generate an Abelian ideal.

\section{Three-level contraction}

The standard contraction procedure can be generalized. Assume the original Lie algebra $\mathfrak{g}$ admits a decomposition into three parts,

$$
\mathfrak{g}=\dot{\mathfrak{i}}_{0} \oplus \mathfrak{i}_{1} \oplus \dot{\mathfrak{t}}_{2},
$$

with the condition 


$$
\left[\mathfrak{i}_{0}, \dot{\mathfrak{t}}_{0}\right] \subset \mathfrak{i}_{0}, \quad\left[\mathfrak{i}_{0}, \dot{\mathfrak{i}}_{1}\right] \subset \mathfrak{i}_{0} \oplus \mathfrak{i}_{1}
$$

If we rescale the generators $\dot{i}_{1}$ and $\dot{\mathfrak{t}}_{2}$ by $c^{-1}$ and $c^{-2}$, respectively,

$$
\dot{\mathrm{j}}_{0}=\dot{\mathfrak{i}}_{0}, \quad \dot{\mathrm{i}}_{1}=\frac{1}{c} \dot{\mathfrak{i}}_{1}, \quad \dot{\mathrm{i}}_{2}=\frac{1}{c^{2}} \dot{\mathfrak{i}}_{2},
$$

the contraction limit $c \rightarrow \infty$ gives a new algebra,

$$
\mathcal{G}(\mathfrak{g})=\dot{\mathrm{j}}_{0} \oplus \dot{\mathrm{j}}_{1} \oplus \dot{\mathrm{j}}_{2},
$$

with the Lie brackets

$$
\begin{array}{ll}
{\left[\dot{\mathrm{j}}_{0}, \dot{\mathrm{j}}_{0}\right] \subset \dot{\mathrm{j}}_{0},} & {\left[\dot{\mathrm{j}}_{0}, \dot{\mathrm{j}}_{1}\right] \subset \dot{\mathrm{j}}_{1},} \\
{\left[\dot{\mathrm{j}}_{0}, \dot{\mathrm{j}}_{2}\right] \subset \dot{\mathrm{j}}_{2},} & {\left[\dot{\mathrm{j}}_{1}, \dot{\mathrm{j}}_{1}\right] \subset \dot{\mathrm{j}}_{2},}
\end{array}
$$

and

$$
\left[\dot{\mathrm{j}}_{1}, \dot{\mathrm{j}}_{2}\right] \subset \varnothing, \quad\left[\dot{\mathrm{j}}_{2}, \dot{\mathrm{j}}_{2}\right] \subset \varnothing .
$$

Note that $\dot{\mathrm{j}}_{2}$ and $\dot{\mathrm{i}}_{1}+\dot{\mathrm{j}}_{2}$ are two different ideals of the contracted Lie algebra. This contraction actually covers all the nonrelativistic limits considered in this paper.

\section{Multilevel contraction}

We can further generalize the contraction procedure by considering arbitrary many "levels," i.e., several integer rescaling dimensions. In the mathematical physics literature, this generalized İnönü-Wigner contraction has been studied in Ref. [33].

Our starting point is again a decomposition of the original algebra

$$
\mathfrak{g}=\mathfrak{i}_{0} \oplus \dot{\mathfrak{i}}_{1} \oplus \cdots \oplus \dot{\mathfrak{i}}_{L},
$$

with the condition

$$
\left[\mathfrak{i}_{m}, \dot{\mathfrak{i}}_{n}\right] \subset \mathfrak{i}_{0} \oplus \cdots \oplus \dot{\mathfrak{i}}_{m+n}, \quad \dot{\mathfrak{i}}_{n>L}=\varnothing
$$

After the rescaling

$$
\dot{\mathfrak{j}}_{k}=\frac{1}{c^{k}} \dot{\mathfrak{t}}_{k},
$$

and the contraction $c \rightarrow \infty$, we obtain a new algebra,

$$
\mathcal{G}(\mathfrak{g})=\dot{\mathrm{j}}_{0} \oplus \dot{\mathrm{j}}_{1} \oplus \cdots \oplus \dot{\mathrm{j}}_{L},
$$

with the Lie brackets

$$
\left[\dot{\mathrm{j}}_{m}, \dot{\mathrm{j}}_{n}\right] \subset \dot{\mathrm{j}}_{m+n}, \quad \dot{\mathrm{j}}_{n>L}=\varnothing .
$$

When the levels of the elements are higher than $L / 2$, their commutators will vanish as the maximum is $L$. At least half of the ideals $\dot{\mathrm{j}}_{n} \oplus \dot{\mathrm{j}}_{n+1} \oplus \cdots \oplus \dot{\mathrm{i}}_{L}$ are Abelian. We need $L>1$ to have a non-Abelian ideal.

Mathematically, the condition (71) is nothing but a filtration structure, for which it is more natural to work with

$$
\mathfrak{f}_{n}=\mathfrak{i}_{0} \oplus \cdots \oplus \mathfrak{i}_{n} .
$$

Then, the decomposition (70) and the condition (71) can be recast as the definition of the filtered Lie algebra:

$\mathfrak{f}_{0} \subset \cdots \subset \mathfrak{f}_{L-1} \subset \mathfrak{f}_{L}=\mathfrak{g}, \quad$ with $\quad\left[\mathfrak{f}_{m}, \mathfrak{f}_{n}\right] \subset \mathfrak{f}_{m+n}$.

For a given filtered (Lie) algebra $\mathfrak{g}$, there exists a graded (Lie) algebra $\mathcal{G}(\mathfrak{g})$ with Eq. (74). The latter is referred to as "associated graded (Lie) algebra."

An example of multilevel contractions can be found in higher-spin algebras. By definition, the vector space of a higher-spin algebra consists of the spaces $\boldsymbol{K}_{s}$ of spin $s$ Killing tensors,

$$
\mathfrak{g}_{\mathrm{HS}}=\bigoplus_{s} \mathfrak{\Re}_{s} .
$$

Each $\boldsymbol{\Re}_{s}$ is generated by the traceless tensor $M_{a_{1} \cdots a_{s-1}, b_{1} \cdots b_{s-1}}$ with the permutation symmetry of a two-row Young diagram $(s-1, s-1)$. The Lie brackets of the higher-spin algebra satisfy

$$
\left[\mathfrak{K}_{s_{1}}, \mathfrak{K}_{s_{2}}\right] \subset \mathfrak{\Re}_{s_{1}+s_{2}-2} \oplus \mathfrak{\Re}_{s_{1}+s_{2}-3} \oplus \cdots
$$

The spins on the right-hand side of Eq. (78) are bounded from above because one cannot construct $(r, r)$ tensors with $r \geq s_{1}+s_{2}-2$ in terms of $M_{\left(s_{1}-1, s_{2}-1\right)}$ and $M_{\left(s_{2}-1, s_{2}-1\right)}{ }^{8}$ Hence, taking $\dot{\mathfrak{i}}_{s-2}=\mathfrak{\mathfrak { R }}_{s}$, one can see that the higher-spin algebra enjoys the filtration structure (71). An unusual point is that a higher-spin algebra is typically infinite dimensional, so it involves infinitely many levels $(L=\infty)$. In three dimensions, one can consider finitedimensional higher-spin algebras. For instance, $s l(N, \mathbb{R})$ is isomorphic to the chiral part of the higher-spin algebras involving spin 2 to $N$. The contracted higher-spin algebra still satisfies all the higher-spin covariance conditions but does not admit an invariant bilinear form. It would be interesting to check whether a central extension of this contracted higher-spin algebra may admit a nondegenerate bilinear form, as in the case of three-dimensional nonrelativistic spin-2 algebras. ${ }^{9}$

\section{Nonrelativistic colored-gravity algebra}

In this subsection, we will study the nonrelativistic limits of the three-dimensional colored-gravity algebra using the

\footnotetext{
${ }^{8}$ The case of $r=s_{1}+s_{2}-2$ is ruled out by the antisymmetry of Lie bracket.

${ }^{9}$ The case of $\operatorname{sl}(3, \mathbb{R})$ was discussed in Ref. [19].
} 
generalized İnönü-Wigner contraction. The guiding principle is that the limiting algebra should have a nondegenerate bilinear form. This nondegeneracy is related to the physical requirement that the kinetic terms are present. ${ }^{10}$ As we have seen before, this condition cannot be met in the chiral sector, so we will work with the full algebra from now on.

We shall define the generators of the nonrelativistic colored-gravity algebras in terms of the relativistic ones and the contraction parameter $c$. For the color-singlet generators, we will use the same contraction ansatz as the doubly extended NH algebra $\mathfrak{g}_{\Lambda}^{++}$, i.e., Eqs. (9), (11), and (12). This choice corresponds to the three-level ansatz:

$$
\begin{aligned}
& \dot{\mathrm{j}}_{0}: \mathcal{Z}+\mathcal{L}_{0}, \quad \tilde{\mathcal{Z}}+\tilde{\mathcal{L}}_{0}, \\
& \dot{\mathrm{j}}_{1}: \frac{\mathcal{L}_{i}}{c}, \quad \frac{\tilde{\mathcal{L}}_{i}}{c}, \\
& \dot{\mathrm{j}}_{2}: \frac{\mathcal{Z}-\mathcal{L}_{0}}{c^{2}}, \quad \frac{\tilde{\mathcal{Z}}-\tilde{\mathcal{L}}_{0}}{c^{2}} .
\end{aligned}
$$

The nonsinglet generators of the colored-gravity algebra should be appended to $\dot{\mathrm{j}}_{0}, \dot{\mathrm{j}}_{1}$, and $\dot{\mathrm{j}}_{2}$ such that the contracted algebra admits a nondegenerate bilinear form. From the symmetry consideration, the bilinear form of the nonsinglet sector generators is determined by that of the singlet sector as

$$
\left\langle a \otimes \mathcal{T}^{I}, b \otimes \mathcal{T}^{J}\right\rangle=\langle a, b\rangle \delta^{I J},
$$

where $a$ and $b$ are elements of $g l(2) \oplus g l(2)$ and their bilinear form is given in Sec. II C. Let us summarize it here for the reader's convenience: due to the associative structure, the bilinear form should be diagonal, i.e., $\langle\mathcal{Z}, \tilde{\mathcal{Z}}\rangle=\gamma=0$, and then we have

$$
\begin{aligned}
\langle\mathcal{Z}, \mathcal{Z}\rangle & =c^{2} \ell+\beta / 2, \quad\left\langle\mathcal{L}_{a}, \mathcal{L}_{b}\right\rangle=\eta_{a b}\left(c^{2} \ell-\beta / 2\right), \\
\langle\tilde{\mathcal{Z}}, \tilde{\mathcal{Z}}\rangle=c^{2} \tilde{\ell}+\tilde{\beta} / 2, & \left\langle\tilde{\mathcal{L}}_{a}, \tilde{\mathcal{L}}_{b}\right\rangle=\eta_{a b}\left(c^{2} \tilde{\ell}-\tilde{\beta} / 2\right) .
\end{aligned}
$$

As one can see, they all scale as $c^{2}$ and hence diverge unless we rescale them appropriately. About the spatial part of nonsinglet generators, we take the analogous rescaling as the singlet ones,

$$
\dot{\mathrm{j}}_{1}: \frac{\mathcal{L}_{i} \otimes \mathcal{T}^{I}}{c}, \quad \frac{\tilde{\mathcal{L}}_{i} \otimes \mathcal{T}^{I}}{c},
$$

and hence these generators belong to $\dot{\mathrm{i}}_{1}$. About the remaining generators, there are a few consistent possibilities. In the classification, an important guideline is the fact that $\dot{j}_{0}$ must form a subalgebra for a consistent contraction.

\footnotetext{
${ }^{10}$ In a more general setting, we may consider degenerate bilinear form. Then, some fields will not have kinetic terms and only appear in the cubic terms.
}

Moreover, due to the presence of $s u(N)$ part, the $g l(2) \oplus g l(2)$ part of the nonsinglet elements in $\dot{\mathrm{j}}_{0}$ should be closed under the anticommutator. Let us write the nonsinglet part of $\dot{\mathfrak{j}}_{0}$ as $\mathfrak{A} \otimes \mathcal{T}^{I}$; then, $\mathfrak{A}$ should satisfy

$$
\{\mathfrak{A}, \mathfrak{A}\} \subset \mathfrak{A},
$$

where $\mathfrak{A}$ is a subspace of $g l(2) \oplus g l(2)$. With the above condition, let us examine what the possible candidates for the $\mathfrak{A}$ elements are. The first guess is the combinations analogous to the singlet sector,

$$
\left(\mathcal{Z}+\mathcal{L}_{0}\right) \otimes \mathcal{T}^{I}, \quad\left(\tilde{\mathcal{Z}}+\tilde{\mathcal{L}}_{0}\right) \otimes \mathcal{T}^{I},
$$

but one can easily see that neither $\mathcal{Z}+\mathcal{L}_{0}$ nor $\tilde{\mathcal{Z}}+\tilde{\mathcal{L}}_{0}$ is closed under the anticommutator. In addition, we cannot consider $\mathcal{Z}$ or $\tilde{\mathcal{Z}}$ because its bilinear form will diverge. In fact, the only possible way to have a finite bilinear form in the $\dot{\mathrm{j}}_{0}$ part - that is, without rescaling the generators-is to cancel the $c^{2}$ terms; for instance, $\left\langle\mathcal{Z}+\mathcal{L}_{0}, \mathcal{Z}+\mathcal{L}_{0}\right\rangle=$ $\langle\mathcal{Z}, \mathcal{Z}\rangle+\left\langle\mathcal{L}_{0}, \mathcal{L}_{0}\right\rangle=\left(c^{2} \ell+\beta / 2\right)-\left(c^{2} \ell-\beta / 2\right)=\beta \quad$ in the singlet sector. Noticing this, we can see

$$
\dot{\mathrm{j}}_{0}:(\mathcal{Z}+\tilde{\mathcal{Z}}) \otimes \mathcal{T}^{I}
$$

has a finite bilinear form, $\langle\mathcal{Z}+\tilde{\mathcal{Z}}, \mathcal{Z}+\tilde{\mathcal{Z}}\rangle=\frac{\beta+\tilde{\beta}}{2}$, and it is closed under the anticommutator: $(\mathcal{Z}+\tilde{\mathcal{Z}})^{2}=\mathcal{Z}+\tilde{\mathcal{Z}}$. The similar combination $\mathcal{Z}-\tilde{\mathcal{Z}}$ has a finite bilinear form but is not closed. For the nondegeneracy of the bilinear form, the choice (85) should be accompanied by

$$
\dot{\mathrm{j}}_{2}: \frac{(\mathcal{Z}-\tilde{\mathcal{Z}}) \otimes \mathcal{T}^{I}}{c^{2}} .
$$

Now, there remain only two generators, $\mathcal{L}_{0}$ and $\tilde{\mathcal{L}}_{0}$, and there are two nontrivial choices:

$\dot{\mathrm{j}}_{0}:\left(\mathcal{L}_{0} \pm \tilde{\mathcal{L}}_{0}\right) \otimes \mathcal{T}^{I}, \quad \dot{\mathrm{i}}_{2}: \frac{\left(\mathcal{L}_{0} \mp \tilde{\mathcal{L}}_{0}\right) \otimes \mathcal{T}^{I}}{c^{2}}$.

Note that both signs give consistent contractions. There also exist rather trivial options: i) the entire nonsinglet generators are in $\dot{\mathfrak{i}}_{1}$, and ii) we have Eqs. (85) and (86), but $\mathcal{L}_{0} \otimes \mathcal{T}^{I}$ and $\tilde{\mathcal{L}}_{0} \otimes \mathcal{T}^{I}$ are in $\dot{\mathfrak{i}}_{1}$. The latter two cases are mathematically consistent options, but they treat the colordecorated isometry generators $\mathcal{L}_{a} \otimes \mathcal{T}^{I}$ in the Lorentzinvariant manner, and hence they are unnatural for the interpretation as nonrelativistic physics. In the following, we consider only the "nontrivial" cases. See Appendix C for the other two cases. Another implicit assumption is that the $s u(N)$ symmetry is not broken in the nonrelativistic limits. There will be more possibilities if we relax this assumption. 


\section{CHERN-SIMONS FORMULATION}

In this section, we derive the actions of the nonrelativistic colored-gravity theories. There are two ways to compute these nonrelativistic actions. The first way is to directly evaluate the Chern-Simons actions of which the gauge fields take values in the nonrelativistic gauge algebras. The second way is to take the nonrelativistic limits of the relativistic Chern-Simons action according to the contractions. We will use the first method in the current section and discuss the second one in Appendix B.

\section{A. Gauge algebras}

Before starting our discussion, let us summarize the algebraic structure of nonrelativistic colored-gravity symmetry. For a more clear physical interpretation, we use " $\mathcal{J}, \mathcal{P}$ " bases rather than the chiral and antichiral bases. Since the singlet sector is identical to the doubly extended $\mathrm{NH}$ algebra, we directly start with the nonsinglet ones. The contractions we have considered in the previous section are

$$
\begin{array}{rlrl}
C^{I} & =\mathcal{Z}_{\mathcal{J}} \otimes \mathcal{T}^{I}, & D^{I}=\frac{1}{c^{2}} \mathcal{Z}_{\mathcal{P}} \otimes \mathcal{T}^{I}, \\
\boldsymbol{G}_{i}^{I}=\frac{1}{c} \mathcal{J}_{i} \otimes \mathcal{T}^{I}, & \boldsymbol{P}_{i}^{I}=\frac{1}{c} \mathcal{P}_{i} \otimes \mathcal{T}^{I} .
\end{array}
$$

These generators transform covariantly under the $\mathrm{NH}$ symmetry as

$$
\begin{aligned}
{\left[J, \boldsymbol{G}_{i}^{I}\right]=2 \epsilon_{i j} \boldsymbol{G}_{j}^{I}, } & {\left[J, \boldsymbol{P}_{i}^{I}\right]=2 \epsilon_{i j} \boldsymbol{P}_{j}^{I}, } \\
{\left[H, \boldsymbol{G}_{i}^{I}\right]=2 \epsilon_{i j} \boldsymbol{P}_{j}^{I}, } & {\left[H, \boldsymbol{P}_{i}^{I}\right]=-2 \Lambda \epsilon_{i j} \boldsymbol{G}_{j}^{I}, }
\end{aligned}
$$

and they have nontrivial Lie brackets among themselves:

$$
\begin{array}{ll}
{\left[\boldsymbol{C}^{I}, \boldsymbol{C}^{J}\right]=2 i f^{I J}{ }_{K} \boldsymbol{C}^{K},} & {\left[\boldsymbol{C}^{I}, \boldsymbol{D}^{J}\right]=2 i f^{I J}{ }_{K} \boldsymbol{D}^{K},} \\
{\left[\boldsymbol{C}^{I}, \boldsymbol{G}_{i}^{J}\right]=2 i f^{I J}{ }_{K} \boldsymbol{G}_{i}^{K},} & {\left[\boldsymbol{C}^{I}, \boldsymbol{P}_{i}^{J}\right]=2 i f^{I J}{ }_{K} \boldsymbol{P}_{i}^{K} .}
\end{array}
$$

Besides $\boldsymbol{C}^{I}, \boldsymbol{D}^{I}, \boldsymbol{G}_{i}^{I}$, and $\boldsymbol{P}_{i}^{I}$, we also have the temporal and central generators. The two possibilities of the sign in Eq. (87) are translated into the following two cases:

$$
\text { Type A : } \boldsymbol{J}^{I}=\mathcal{J}_{0} \otimes \mathcal{T}^{I}, \quad \boldsymbol{M}^{I}=\frac{1}{c^{2}} \mathcal{P}_{0} \otimes \mathcal{T}^{I},
$$

Type B : $\boldsymbol{H}^{I}=\mathcal{P}_{0} \otimes \mathcal{T}^{I}, \quad S^{I}=\frac{1}{c^{2}} \mathcal{J}_{0} \otimes \mathcal{T}^{I}$.

Only one of the two central generators in the doubly extended NH algebra admits color decoration. Let us present the Lie brackets of the two cases.

\section{Type A}

The nonzero commutators involving one singlet and one nonsinglet are

$$
\begin{aligned}
{\left[\boldsymbol{J}^{I}, G_{i}\right] } & =2 \epsilon_{i j} \boldsymbol{G}_{j}^{I}, \quad\left[\boldsymbol{J}^{I}, P_{i}\right]=2 \epsilon_{i j} \boldsymbol{P}_{j}^{I}, \\
{\left[\boldsymbol{G}_{i}^{I}, P_{j}\right] } & =\left[G_{i}, \boldsymbol{P}_{j}^{I}\right]=-2 \epsilon_{i j} \boldsymbol{M}^{I} .
\end{aligned}
$$

The nonzero commutators involving only the nonsinglet sectors are

$$
\begin{aligned}
{\left[\boldsymbol{C}^{I}, \boldsymbol{J}^{J}\right] } & =2 i f^{I J}{ }_{K} \boldsymbol{J}^{K}, \\
{\left[\boldsymbol{C}^{I}, \boldsymbol{M}^{J}\right] } & =\left[\boldsymbol{D}^{I}, \boldsymbol{J}^{J}\right]=2 i f^{I J}{ }_{K} \boldsymbol{M}^{K}, \\
{\left[\boldsymbol{J}^{I}, \boldsymbol{J}^{J}\right] } & =-2 i f^{I J}{ }_{K} \boldsymbol{C}^{K}, \quad\left[\boldsymbol{J}^{I}, \boldsymbol{M}^{J}\right]=-2 i f^{I J}{ }_{K} \boldsymbol{D}^{K}, \\
{\left[\boldsymbol{J}^{I}, \boldsymbol{G}_{i}^{J}\right] } & =2 \epsilon_{i j}\left(\frac{1}{N} \delta^{I J} G_{j}+g^{I J}{ }_{K} \boldsymbol{G}_{j}^{K}\right), \\
{\left[\boldsymbol{J}^{I}, \boldsymbol{P}_{i}^{J}\right] } & =2 \epsilon_{i j}\left(\frac{1}{N} \delta^{I J} P_{j}+g^{I J}{ }_{K} \boldsymbol{P}_{j}^{K}\right), \\
{\left[\boldsymbol{G}_{i}^{I}, \boldsymbol{G}_{j}^{J}\right] } & =-\frac{1}{\Lambda}\left[\boldsymbol{P}_{i}^{I}, \boldsymbol{P}_{j}^{J}\right]=\frac{2}{N} \epsilon_{i j} \delta^{I J} S, \\
{\left[\boldsymbol{G}_{i}^{I}, \boldsymbol{P}_{j}^{J}\right] } & =\frac{2}{N} \epsilon_{i j} \delta^{I J} M-2 \epsilon_{i j} g^{I J}{ }_{K} \boldsymbol{M}^{K}+2 i \delta_{i j} f^{I J}{ }_{K} \boldsymbol{D}^{K} .
\end{aligned}
$$

\section{Type B}

The nonzero commutators containing one singlet and one nonsinglet sector are

$$
\begin{aligned}
{\left[\boldsymbol{H}^{I}, G_{i}\right] } & =2 \epsilon_{i j} \boldsymbol{P}_{j}^{I}, \quad\left[\boldsymbol{H}^{I}, P_{i}\right]=-2 \Lambda \epsilon_{i j} \boldsymbol{G}_{j}^{I}, \\
{\left[G_{i}, \boldsymbol{G}_{j}^{I}\right] } & =-\frac{1}{\Lambda}\left[P_{i}, \boldsymbol{P}_{j}^{I}\right]=-2 \epsilon_{i j} \boldsymbol{S}^{I} .
\end{aligned}
$$

The nonzero Lie brackets involving only the nonsinglet sector are

$$
\begin{aligned}
{\left[\boldsymbol{C}^{I}, \boldsymbol{H}^{J}\right] } & =2 i f^{I J}{ }_{K} \boldsymbol{H}^{K}, \\
{\left[\boldsymbol{C}^{I}, \boldsymbol{S}^{J}\right] } & =-\frac{1}{\Lambda}\left[\boldsymbol{D}^{I}, \boldsymbol{H}^{J}\right]=2 i f^{I J}{ }_{K} \boldsymbol{S}^{K}, \\
{\left[\boldsymbol{H}^{I}, \boldsymbol{H}^{J}\right] } & =2 i \Lambda f^{I J}{ }_{K} \boldsymbol{C}^{K}, \quad\left[\boldsymbol{S}^{I}, \boldsymbol{H}^{J}\right]=-2 i f^{I J}{ }_{K} \boldsymbol{D}^{K}, \\
{\left[\boldsymbol{H}^{I}, \boldsymbol{G}_{i}^{J}\right] } & =2 \epsilon_{i j}\left(\frac{1}{N} \delta^{I J} P_{j}+g^{I J}{ }_{K} \boldsymbol{P}_{j}^{K}\right), \\
{\left[\boldsymbol{H}^{I}, \boldsymbol{P}_{i}^{J}\right] } & =-2 \Lambda \epsilon_{i j}\left(\frac{1}{N} \delta^{I J} G_{j}+g^{I J}{ }_{K} \boldsymbol{G}_{j}^{K}\right), \\
{\left[\boldsymbol{G}_{i}^{I}, \boldsymbol{G}_{j}^{J}\right] } & =-\frac{1}{\Lambda}\left[\boldsymbol{P}_{i}^{I}, \boldsymbol{P}_{j}^{J}\right]=\frac{2}{N} \epsilon_{i j} \delta^{I J} S-2 \epsilon_{i j} g^{I J}{ }_{K} \boldsymbol{S}^{J}, \\
{\left[\boldsymbol{G}_{i}^{I}, \boldsymbol{P}_{j}^{J}\right] } & =\frac{2}{N} \epsilon_{i j} \delta^{I J} M+2 i \delta_{i j} f^{I J}{ }_{K} \boldsymbol{D}^{K} .
\end{aligned}
$$




\section{Bilinear form}

Let us also remind the reader that the invariant bilinear form reads

$$
\begin{gathered}
\langle J, M\rangle=\langle S, H\rangle=1, \quad\left\langle P_{i}, G_{j}\right\rangle=\delta_{i j}, \\
\left\langle\boldsymbol{C}^{I}, \boldsymbol{D}^{J}\right\rangle=\delta^{I J}, \quad\left\langle\boldsymbol{P}_{i}^{I}, \boldsymbol{G}_{j}^{J}\right\rangle=\delta_{i j} \delta^{I J},
\end{gathered}
$$

and

$$
\left\langle\boldsymbol{J}^{I}, \boldsymbol{M}^{J}\right\rangle=-\delta^{I J}, \quad\left\langle\boldsymbol{H}^{I}, \boldsymbol{S}^{J}\right\rangle=-\delta^{I J},
$$

for type A and B, respectively. Here, we took the choice $\beta=\tilde{\beta}=\gamma=0$ for simplicity.

\section{B. Actions}

Now, we consider the Chern-Simons actions of the nonrelativistic colored gravities. The 1 -form gauge fields are

Type A:

$$
\begin{aligned}
\mathcal{A}= & j J+s S+h H+m M+\Omega^{i} G_{i}+E^{i} P_{i} \\
& +\sigma_{I} \boldsymbol{C}^{I}+\varsigma_{I} \boldsymbol{D}^{I}+\vartheta_{I} \boldsymbol{J}^{I}+\varpi_{I} \boldsymbol{M}^{I} \\
& +\theta_{I}^{i} \boldsymbol{G}_{i}^{I}+\pi_{I}^{i} \boldsymbol{P}_{i}^{I},
\end{aligned}
$$

Type B :

$$
\begin{aligned}
\mathcal{A}= & j J+s S+h H+m M+\Omega^{i} G_{i}+E^{i} P_{i} \\
& +\sigma_{I} \boldsymbol{C}^{I}+\varsigma_{I} \boldsymbol{D}^{I}+\vartheta_{I} \boldsymbol{S}^{I}+\varpi_{I} \boldsymbol{H}^{I} \\
& +\theta_{I}^{i} \boldsymbol{G}_{i}^{I}+\pi_{I}^{i} \boldsymbol{P}_{i}^{I},
\end{aligned}
$$

which correspond to the nonrelativistic colored-gravity algebras constructed in Sec. III C. It is now sufficient to plug the above into the Chern-Simons action,

$$
S=\frac{\kappa}{4 \pi} \int\langle\mathcal{A} \wedge d \mathcal{A}\rangle+\frac{2}{3}\langle\mathcal{A} \wedge \mathcal{A} \wedge \mathcal{A}\rangle
$$

In terms of the component fields, this action can be decomposed into several pieces:

$$
S=S_{\text {gravity }}+S_{\text {gauge }}+S_{\text {matter }} \text {. }
$$

Here, $S_{\text {gravity }}$ is the usual uncolored nonrelativistic action,

$$
\begin{aligned}
S_{\text {gravity }}= & \frac{\kappa N}{2 \pi} \int E^{i} \wedge \nabla \Omega_{i}+m \wedge d j \\
& +h \wedge\left[d s+\epsilon_{i j}\left(\Omega^{i} \wedge \Omega^{j}-\Lambda E^{i} \wedge E^{j}\right)\right],
\end{aligned}
$$

where the covariant derivative $\nabla$ acts on "spatial" 1-form $\omega_{i}$ as

$$
\nabla \omega_{i}=d \omega_{i}+\epsilon_{i j}\left(\omega^{j} \wedge j-j \wedge \omega^{j}\right) .
$$

About the other pieces of the action, it is convenient to introduce the $s u(N)$-valued fields as

$$
\begin{array}{rrr}
\boldsymbol{\sigma}=\sigma_{I} \mathcal{T}^{I}, & \boldsymbol{\mathcal { S }}=\varsigma_{I} \mathcal{T}^{I}, & \boldsymbol{\vartheta}=\vartheta_{I} \mathcal{T}^{I}, \\
\boldsymbol{\varpi}=\varpi_{I} \mathcal{T}^{I}, & \boldsymbol{\theta}^{i}=\theta_{I}^{i} \mathcal{T}^{I}, & \boldsymbol{\pi}^{i}=\pi_{I}^{i} \mathcal{T}^{I}
\end{array}
$$

and write the action in terms of $\mathrm{Tr}$, the trace in the adjoint representation. Then, the gauge field part $S_{\text {gauge }}$ takes the simple form

$$
S_{\text {gauge }}=\frac{\kappa}{2 \pi} \int \operatorname{Tr}[\boldsymbol{\varsigma} \wedge(d \boldsymbol{\sigma}+\boldsymbol{\sigma} \wedge \boldsymbol{\sigma})] .
$$

The matter field part $S_{\text {matter }}$ depends whether we consider the type A or B:

$$
S_{\text {matter }}=S_{\text {matter }, 0}+S_{\text {type A } / \text { B }} \text {. }
$$

The common part $S_{\text {matter }, 0}$ is

$$
\begin{aligned}
S_{\text {matter }, 0}= & \frac{\kappa}{2 \pi} \int \operatorname{Tr}\left[-\boldsymbol{\vartheta} \wedge D \boldsymbol{\varpi}+\boldsymbol{\theta}^{i} \wedge D \boldsymbol{\pi}_{i}\right. \\
& \left.+\epsilon_{i j} h \wedge\left(\boldsymbol{\theta}^{i} \wedge \boldsymbol{\theta}^{j}-\Lambda \boldsymbol{\pi}^{i} \wedge \boldsymbol{\pi}^{j}\right)\right],
\end{aligned}
$$

where the covariant derivative $D$ acts on the $s u(N)$-valued 1 -form $\omega$ as

$$
D \omega=\nabla \omega+\sigma \wedge \omega+\omega \wedge \boldsymbol{\sigma},
$$

and $\omega$ can have spatial indices.

Finally, the last piece that depends on the type of the nonrelativistic limit is

$$
\begin{aligned}
S_{\text {type A }}= & \frac{\kappa}{2 \pi} \int \operatorname{Tr}\left[-\boldsymbol{\varsigma} \wedge \boldsymbol{\vartheta} \wedge \boldsymbol{\vartheta}+2 \epsilon_{i j} \boldsymbol{\vartheta} \wedge\left(\boldsymbol{\pi}^{i} \wedge \Omega^{j}\right.\right. \\
& \left.\left.+\boldsymbol{\theta}^{i} \wedge E^{j}+\boldsymbol{\theta}^{i} \wedge \boldsymbol{\pi}^{j}\right)\right], \\
S_{\mathrm{type} \mathrm{B}}= & \frac{\kappa}{2 \pi} \int \operatorname{Tr}\left[\Lambda \boldsymbol{\varsigma} \wedge \boldsymbol{\varpi} \wedge \boldsymbol{\varpi}+\epsilon_{i j} \varpi \wedge\left(2 \boldsymbol{\theta}^{i} \wedge \Omega^{j}\right.\right. \\
& \left.\left.-2 \Lambda \boldsymbol{\pi}^{i} \wedge E^{j}+\boldsymbol{\theta}^{i} \wedge \boldsymbol{\theta}^{j}-\Lambda \boldsymbol{\pi}^{i} \wedge \boldsymbol{\pi}^{j}\right)\right] .
\end{aligned}
$$

\section{CONCLUSION}

In this work, we have studied the nonrelativistic limits of three-dimensional colored gravity in terms of generalized İnönü-Wigner contractions. Let us conclude our work with a brief summary of what has been done. In Sec. II, we revisited the three-dimensional nonrelativistic isometry algebras and their bilinear forms. We reviewed various central extensions of the Galilei and Newton-Hooke algebras. Then, we discussed their chiral decompositions, how 
they arise from the contractions of relativistic algebras, and the issue of invariant bilinear forms. In Sec. III, we explained the obstruction in the chiral decompositions of nonrelativistic colored gravity and constructed the consistent nonrelativistic colored-gravity algebras using generalized İnönü-Wigner contractions. In Sec. IV, we derived the nonrelativistic colored-gravity actions in the Chern-Simons formulation using the nonrelativistic algebras constructed in Sec. III.

\section{ACKNOWLEDGMENTS}

We thank Karapet Mkrtchyan for useful discussions in the early stages of the work. W. L. wants to thank Yang Lei for useful discussions. This work was supported by a grant from Kyung Hee University in 2017 (Grant No. KHU-20171742).

\section{APPENDIX A: ASSOCIATIVE STRUCTURE OF RELATIVISTIC SYMMETRY}

If we identify the identities $\mathcal{I}$ and $\tilde{\mathcal{I}}$ with $\mathcal{Z}$ and $\tilde{\mathcal{Z}}$, the associative products of $g l(2) \oplus g l(2)$ generators are

$$
\begin{aligned}
& \mathcal{J}_{a} \mathcal{J}_{b}=-\frac{1}{\Lambda} \mathcal{P}_{a} \mathcal{P}_{b}=\eta_{a b} \mathcal{Z}_{\mathcal{J}}+\epsilon_{a b}{ }^{c} \mathcal{J}_{c} \\
& \mathcal{J}_{a} \mathcal{P}_{b}=\eta_{a b} \mathcal{Z}_{\mathcal{P}}+\epsilon_{a b}{ }^{c} \mathcal{P}_{c} \\
& \mathcal{Z}_{\mathcal{J}} \mathcal{Z}_{\mathcal{J}}=\mathcal{Z}_{\mathcal{J}}, \quad \mathcal{Z}_{\mathcal{J}} \mathcal{Z}_{\mathcal{P}}=\mathcal{Z}_{\mathcal{P}} \mathcal{Z}_{\mathcal{J}}=\mathcal{Z}_{\mathcal{P}}, \\
& \mathcal{Z}_{\mathcal{P}} \mathcal{Z}_{\mathcal{P}}=-\Lambda \mathcal{Z}_{\mathcal{J}}, \\
& \mathcal{J}_{a} \mathcal{Z}_{\mathcal{J}}=\mathcal{Z}_{\mathcal{J}} \mathcal{J}_{a}=\mathcal{J}_{a}, \quad \mathcal{J}_{a} \mathcal{Z}_{\mathcal{P}}=\mathcal{Z}_{\mathcal{P}} \mathcal{J}_{a}=\mathcal{P}_{a}, \\
& \mathcal{P}_{a} \mathcal{Z}_{\mathcal{J}}=\mathcal{Z}_{\mathcal{J}} \mathcal{P}_{a}=\mathcal{P}_{a}, \quad \mathcal{P}_{a} \mathcal{Z}_{\mathcal{P}}=\mathcal{Z}_{\mathcal{P}} \mathcal{P}_{a}=-\Lambda \mathcal{J}_{a}
\end{aligned}
$$

This associative extension of the relativistic isometry algebra can be realized as a tensor product,

$$
\begin{aligned}
& \mathcal{J}_{a}=\mathcal{L}_{a} \otimes I, \quad \mathcal{P}_{a}=\mathcal{L}_{a} \otimes \psi, \\
& Z_{\mathcal{J}}=\mathcal{I} \otimes I,
\end{aligned}
$$

with

$$
\begin{gathered}
\mathcal{L}_{a} \mathcal{L}_{b}=\eta_{a b} \mathcal{I}+\epsilon_{a b}{ }^{c} \mathcal{L}_{c}, \quad I^{2}=I, \\
\psi^{2}=-\Lambda I, \quad I \psi=\psi I=\psi .
\end{gathered}
$$

The chiral decompositions of the relativistic and the nonrelativistic algebras are due to the existence of projectors,

$P_{L}=\frac{1}{2}\left(I+\frac{1}{\sqrt{-\Lambda}} \psi\right), \quad P_{R}=\frac{1}{2}\left(I-\frac{1}{\sqrt{-\Lambda}} \psi\right)$, with

$P_{L}^{2}=P_{L}, \quad P_{R}^{2}=P_{R}, \quad P_{L} P_{R}=P_{R} P_{L}=0$.

\section{APPENDIX B: CONTRACTION AT ACTION LEVEL}

The 1-form gauge field (117) or (118) of the nonrelativistic colored gravities should match with the relativistic one,

$$
\begin{aligned}
\mathcal{A}= & \left(A \mathcal{Z}+\tilde{A} \tilde{\mathcal{Z}}+\omega^{a} \mathcal{J}_{a}+e^{a} \mathcal{P}_{a}\right) \otimes I \\
& +\left(A_{I} \mathcal{Z}+\tilde{A_{I}} \tilde{\mathcal{Z}}+\tau_{I}^{a} \mathcal{J}_{a}+\chi_{I}^{a} \mathcal{P}_{a}\right) \otimes \boldsymbol{T}^{I} .
\end{aligned}
$$

In this way, we are able to identify the relations between relativistic and nonrelativistic fields. The singlet part is

$$
\begin{aligned}
j & =\frac{1}{4}(A+\tilde{A})+\frac{1}{2} \omega^{0}, \quad s=\frac{c^{2}}{2}(A+\tilde{A})-c^{2} \omega^{0}, \\
h & =\frac{1}{4 \sqrt{-\Lambda}}(A-\tilde{A})+\frac{1}{2} e^{0}, \quad m=\frac{c^{2}}{2 \sqrt{-\Lambda}}(A-\tilde{A})-c^{2} e^{0}, \\
\Omega^{i} & =c \omega^{i}, \quad E^{i}=c e^{i} .
\end{aligned}
$$

The nonrelativistic $s u(N)$ gauge fields are

$$
\sigma_{I}=\frac{1}{2}\left(A_{I}+\tilde{A}_{I}\right), \quad \varsigma_{I}=\frac{c^{2}}{2 \sqrt{-\Lambda}}\left(A_{I}-\tilde{A}_{I}\right),
$$

and the spatial components of the matter fields are

$$
\theta_{I}^{i}=c \tau_{I}^{i}, \quad \pi_{I}^{i}=c \chi_{I}^{i} .
$$

Finally, the temporal components of the matter fields are defined as

$$
\begin{array}{lr}
\text { Type A: } \vartheta_{I}=\tau_{I}^{0}, & \varpi_{I}=c^{2} \chi_{I}^{0}, \\
\text { Type B : } \vartheta_{I}=c^{2} \tau_{I}^{0}, & \varpi_{I}=\chi_{I}^{0} .
\end{array}
$$

Substituting the relativistic fields with the nonrelativistic ones, we can derive the nonrelativistic actions in Sec. IV B from the relativistic colored-gravity action by multiplying the action with $c^{2}$ and taking the limit $c \rightarrow \infty$.

\section{APPENDIX C: OTHER CONTRACTIONS OF COLORED-GRAVITY ALGEBRA}

In Sec. III C, we mentioned two other cases of nonrelativistic limits of the colored-gravity algebra. In the first case, we treat all the nonsinglet generators as the level-1 
elements, whereas in the second case, we have additional level-0 and level-2 elements, Eqs. (85) and (86).

In the first case, the nonrelativistic colored generators are defined as

$$
\begin{aligned}
\boldsymbol{C}^{I} & =\frac{1}{c} \mathcal{Z}_{\mathcal{J}} \otimes \mathcal{T}^{I}, & \boldsymbol{D}^{I} & =\frac{1}{c} \mathcal{Z}_{\mathcal{P}} \otimes \mathcal{T}^{I}, \\
\boldsymbol{J}^{I} & =\frac{1}{c} \mathcal{J}_{0} \otimes \mathcal{T}^{I}, & \boldsymbol{H}^{I} & =\frac{1}{c} \mathcal{P}_{0} \otimes \mathcal{T}^{I}, \\
\boldsymbol{G}_{i}^{I} & =\frac{1}{c} \mathcal{J}_{i} \otimes \mathcal{T}^{I}, & \boldsymbol{P}_{i}^{I} & =\frac{1}{c} \mathcal{P}_{i} \otimes \mathcal{T}^{I} .
\end{aligned}
$$

In the nonrelativistic limit, the nonzero commutators involving colored generators are

$$
\begin{aligned}
{\left[J, \boldsymbol{G}_{i}^{I}\right] } & =-\frac{1}{\Lambda}\left[H, \boldsymbol{P}_{i}^{I}\right]=2 \epsilon_{i j} \boldsymbol{G}_{j}^{I}, \\
{\left[J, \boldsymbol{P}_{i}^{I}\right] } & =\left[H, \boldsymbol{G}_{i}^{I}\right]=2 \epsilon_{i j} \boldsymbol{P}_{j}^{I}, \\
{\left[\boldsymbol{G}_{i}^{I}, \boldsymbol{G}_{j}^{J}\right] } & =-\frac{1}{\Lambda}\left[\boldsymbol{P}_{i}^{I}, \boldsymbol{P}_{j}^{J}\right]=\frac{2}{N} \epsilon_{i j} \delta^{I J} S, \\
{\left[\boldsymbol{G}_{i}^{I}, \boldsymbol{P}_{j}^{J}\right] } & =\frac{2}{N} \epsilon_{i j} \delta^{I J} M .
\end{aligned}
$$

The relations between relativistic and nonrelativistic fields are

$\sigma_{I}=\frac{c}{2}\left(A_{I}+\tilde{A_{I}}\right), \quad \varsigma_{I}=\frac{c}{2 \sqrt{-\Lambda}}\left(A_{I}-\tilde{A}_{I}\right)$,

$\vartheta_{I}=c \tau_{I}^{0}, \quad \varpi_{I}=c \chi_{I}^{0}, \quad \theta_{I}^{i}=c \tau_{I}^{i}, \quad \pi_{I}^{i}=c \chi_{I}^{i}$.

The nonrelativistic action is again given as Eq. (120). $S_{\text {gravity }}$ is the same as Eq. (121), whereas $S_{\text {gauge }}$ and $S_{\text {matter }}$ are given by

$$
\begin{aligned}
S_{\text {gauge }}= & \frac{\kappa}{2 \pi} \int \operatorname{Tr}(\boldsymbol{\varsigma} \wedge d \boldsymbol{\sigma}), \\
S_{\text {matter }}= & \frac{\kappa}{2 \pi} \int \operatorname{Tr}\left[-\boldsymbol{\vartheta} \wedge d \boldsymbol{\varpi}+\boldsymbol{\theta}^{i} \wedge \nabla \boldsymbol{\pi}_{i}\right. \\
& \left.+\epsilon_{i j} h \wedge\left(\boldsymbol{\theta}^{i} \wedge \boldsymbol{\theta}^{j}-\Lambda \boldsymbol{\pi}^{i} \wedge \boldsymbol{\pi}^{j}\right)\right] .
\end{aligned}
$$

In the second case, the level-0 and level- 2 sectors contain $\mathcal{Z}_{\mathcal{J}} \otimes \mathcal{T}^{I}$ and $\mathcal{Z}_{\mathcal{P}} \otimes \mathcal{T}^{I}$, respectively. The nonrelativistic generators are defined as

$$
\begin{aligned}
\boldsymbol{C}^{I} & =\mathcal{Z}_{\mathcal{J}} \otimes \mathcal{T}^{I}, & \boldsymbol{D}^{I} & =\frac{1}{c^{2}} \mathcal{Z}_{\mathcal{P}} \otimes \mathcal{T}^{I}, \\
\boldsymbol{J}^{I} & =\frac{1}{c} \mathcal{J}_{0} \otimes \mathcal{T}^{I}, & \boldsymbol{H}^{I} & =\frac{1}{c} \mathcal{P}_{0} \otimes \mathcal{T}^{I} . \\
\boldsymbol{G}_{i}^{I} & =\frac{1}{c} \mathcal{J}_{i} \otimes \mathcal{T}^{I}, & \boldsymbol{P}_{i}^{I} & =\frac{1}{c} \mathcal{P}_{i} \otimes \mathcal{T}^{I} .
\end{aligned}
$$

The nonzero Lie brackets involving the colored generators are

$$
\begin{aligned}
{\left[J, \boldsymbol{G}_{i}^{I}\right] } & =-\frac{1}{\Lambda}\left[H, \boldsymbol{P}_{i}^{I}\right]=2 \epsilon_{i j} \boldsymbol{G}_{j}^{I}, \\
{\left[J, \boldsymbol{P}_{i}^{I}\right] } & =\left[H, \boldsymbol{G}_{i}^{I}\right]=2 \epsilon_{i j} \boldsymbol{P}_{j}^{I}, \\
{\left[\boldsymbol{C}^{I}, \boldsymbol{C}^{J}\right] } & =2 i f^{I J}{ }_{K} \boldsymbol{C}^{K}, \quad\left[\boldsymbol{C}^{I}, \boldsymbol{D}^{J}\right]=2 i f^{I J}{ }_{K} \boldsymbol{D}^{K}, \\
{\left[\boldsymbol{C}^{I}, \boldsymbol{J}^{J}\right] } & =2 i f^{I J}{ }_{K} \boldsymbol{J}^{K}, \quad\left[\boldsymbol{C}^{I}, \boldsymbol{H}^{J}\right]=2 i f^{I J}{ }_{K} \boldsymbol{H}^{K}, \\
{\left[\boldsymbol{C}^{I}, \boldsymbol{G}_{i}^{J}\right] } & =2 i f^{I J}{ }_{K} \boldsymbol{G}_{i}^{K}, \quad\left[\boldsymbol{C}^{I}, \boldsymbol{P}_{i}^{J}\right]=2 i f^{I J}{ }_{K} \boldsymbol{P}_{i}^{K}, \\
{\left[\boldsymbol{J}^{I}, \boldsymbol{H}^{J}\right] } & =-2 i f^{I J}{ }_{K} \boldsymbol{D}^{K}, \\
{\left[\boldsymbol{G}_{i}^{I}, \boldsymbol{G}_{j}^{J}\right] } & =-\frac{1}{\Lambda}\left[\boldsymbol{P}_{i}^{I}, \boldsymbol{P}_{j}^{J}\right]=\frac{2}{N} \epsilon_{i j} \delta^{I J} S, \\
{\left[\boldsymbol{G}_{i}^{I}, \boldsymbol{P}_{j}^{J}\right] } & =\frac{2}{N} \epsilon_{i j} \delta^{I J} M+2 i \delta_{i j} f^{I J}{ }_{K} \boldsymbol{D}^{K} .
\end{aligned}
$$

The nonrelativistic fields are related to the relativistic fields by

$$
\begin{aligned}
& \sigma_{I}=\frac{1}{2}\left(A_{I}+\tilde{A}_{I}\right), \quad \varsigma_{I}=\frac{c^{2}}{2 \sqrt{-\Lambda}}\left(A_{I}-\tilde{A}_{I}\right), \\
& \vartheta_{I}=c \tau_{I}^{0}, \quad \varpi_{I}=c \chi_{I}^{0}, \quad \theta_{I}^{i}=c \tau_{I}^{i}, \quad \pi_{I}^{i}=c \chi_{I}^{i} .
\end{aligned}
$$

Again, the action is given by Eq. (120), where $S_{\text {gravity }}$ and $S_{\text {gauge }}$ correspond to Eqs. (121) and (124), while $S_{\text {matter }}$ coincides with $S_{\text {matter }, 0}$ in Eq. (126).
[1] E. Cartan, Sur les variétés à connexion affine et la théorie de la relativité généralisée. (premire partie), Ann. Sci. École Norm. Sup. 40, 325 (1923).
[2] E. Cartan, Sur les variétés à connexion affine et la théorie de la relativité généralisée. (premire partie) (Suite), Ann. Sci. École Norm. Sup. 41, 1 (1924). 
[3] C. Duval, G. Burdet, H. P. Kunzle, and M. Perrin, Bargmann structures and Newton-Cartan theory, Phys. Rev. D 31, 1841 (1985).

[4] X. Bekaert and K. Morand, Connections and dynamical trajectories in generalised Newton-Cartan gravity I. An intrinsic view, J. Math. Phys. (N.Y.) 57, 022507 (2016).

[5] X. Bekaert and K. Morand, Connections and dynamical trajectories in generalised Newton-Cartan gravity II. An ambient perspective, arXiv:1505.03739.

[6] V. Bargmann, Irreducible unitary representations of the Lorentz group, Ann. Math. 48, 568 (1947).

[7] R. Andringa, E. Bergshoeff, S. Panda, and M. de Roo, Newtonian gravity and the Bargmann algebra, Classical Quantum Gravity 28, 105011 (2011).

[8] E. Bergshoeff, J. Rosseel, and T. Zojer, Newton-Cartan (super)gravity as a non-relativistic limit, Classical Quantum Gravity 32, 205003 (2015).

[9] J. M. Levy-Leblond, Galilei Group and Galilean Invariance, Group Theory and Applications (Academic, New York, 1971), Vol. II.

[10] D. R. Grigore, The projective unitary irreducible representations of the Galilei group in $(1+2)$-dimensions, J. Math. Phys. (N.Y.) 37, 460 (1996).

[11] S. K. Bose, The Galilean group in $(2+1)$ space-times and its central extension, Commun. Math. Phys. 169, 385 (1995).

[12] R. Jackiw and V. P. Nair, Anyon spin and the exotic central extension of the planar Galilei group, Phys. Lett. B 480, 237 (2000).

[13] J. Lukierski, P. C. Stichel, and W. J. Zakrzewski, Galilean invariant $(2+1)$-dimensional models with a Chern-Simonslike term and $\mathrm{D}=2$ noncommutative geometry, Ann. Phys. (N.Y.) 260, 224 (1997).

[14] C. Duval and P. A. Horvathy, The 'Peierls substitution' and the exotic Galilei group, Phys. Lett. B 479, 284 (2000).

[15] G. Papageorgiou and B.J. Schroers, A Chern-Simons approach to Galilean quantum gravity in $2+1$ dimensions, J. High Energy Phys. 11 (2009) 009.

[16] G. Papageorgiou and B. J. Schroers, Galilean quantum gravity with cosmological constant and the extended $q$ Heisenberg algebra, J. High Energy Phys. 11 (2010) 020.

[17] E. A. Bergshoeff and J. Rosseel, Three-Dimensional Extended Bargmann Supergravity, Phys. Rev. Lett. 116, 251601 (2016).

[18] J. Hartong, Y. Lei, and N. A. Obers, Nonrelativistic ChernSimons theories and three-dimensional Horava-Lifshitz gravity, Phys. Rev. D 94, 065027 (2016).
[19] E. Bergshoeff, D. Grumiller, S. Prohazka, and J. Rosseel, Three-dimensional spin-3 theories based on general kinematical algebras, J. High Energy Phys. 01 (2017) 114.

[20] J. Hartong, Y. Lei, N. A. Obers, and G. Oling, Zooming in on $\mathrm{AdS}_{3} / \mathrm{CFT}_{2}$ near a BPS Bound, J. High Energy Phys. 05 (2018) 16.

[21] S. Gwak, E. Joung, K. Mkrtchyan, and S. J. Rey, Rainbow valley of colored (anti) de Sitter gravity in three dimensions, J. High Energy Phys. 04 (2016) 055.

[22] S. Gwak, E. Joung, K. Mkrtchyan, and S. J. Rey, Rainbow vacua of colored higher-spin $(\mathrm{A}) \mathrm{dS}_{3}$ gravity, J. High Energy Phys. 05 (2016) 150.

[23] E. Joung, J. Kim, J. Kim, and S. J. Rey, Asymptotic symmetries of colored gravity in three dimensions, J. High Energy Phys. 03 (2018) 104.

[24] N. Boulanger, T. Damour, L. Gualtieri, and M. Henneaux, Inconsistency of interacting, multigraviton theories, Nucl. Phys. B597, 127 (2001).

[25] N. Boulanger, Multigraviton theories: Yes go and no go results, Fortschr. Phys. 50, 858 (2002).

[26] N. Boulanger, M. Henneaux, and P. van Nieuwenhuizen, Conformal (super)gravities with several gravitons, J. High Energy Phys. 01 (2002) 035.

[27] N. Boulanger and L. Gualtieri, An exotic theory of massless spin two fields in three-dimensions, Classical Quantum Gravity 18, 1485 (2001).

[28] S. Deser and R. I. Nepomechie, Anomalous propagation of gauge fields in conformally flat spaces, Phys. Lett. 132B, 321 (1983).

[29] S. Deser and A. Waldron, Partial masslessness of higher spins in (A)dS, Nucl. Phys. B607, 577 (2001).

[30] C. R. Hagen, Second central extension in Galilean covariant field theory, Phys. Lett. B 539, 168 (2002).

[31] C. Duval and P. A. Horvathy, Spin and exotic Galilean symmetry, Phys. Lett. B 547, 306 (2002); Erratum, Phys. Lett. B 588, 228 (2004).

[32] E. Inonu and E. P. Wigner, On the Contraction of groups and their represenations, Proc. Natl. Acad. Sci. U.S.A. 39, 510 (1953).

[33] E. Weimar-Woods, Contractions of Lie algebras: generalized Inönü-Wigner contractions versus graded contractions, J. Math. Phys. (N.Y.) 36, 4519 (1995); E. Weimar-Woods, Contractions generalized Inönü and Wigner contractions and deformations of finite-dimensional Lie algebras, Rev. Math. Phys. 12, 1505 (2000). 